\title{
GEOMETRIC FEATURES EXTRACTION OF A 3D CAD MODEL FOR RECONFIGURABLE MANUFACTURING
}

\author{
C. S. GHADAGE ${ }^{1}$, Dr. B. S. PABLA ${ }^{2} \&$ Dr. S. S. DHAMI ${ }^{3}$ \\ ${ }^{1}$ Lecturer, Mechanical Engineering Department, Govt. Polytechnic, Pune, India \\ ${ }^{2,3}$ Professor, Mechanical Engineering Department, National Institute of Technical Teachers' Training and Research,
}

Chandigarh, India

\begin{abstract}
Reconfigurable manufacturing systems are planned to allow the use of a variable number of spindles so as to allow meeting the machining requirements of the different batches of components in a single setup and thereby facilitating faster machining and better correlation between the different geometric surfaces. Computer aided process planning is a vital link which gives the complete process plan that can be further used to calculate the number of spindles required to machine the component in a single setup and the planes in which these spindles have to be installed. For Computer aided process planning, the design features have to be converted into manufacturing features for further processing. The CAD information in neutral design data format can be used most effectively for further stages in the product manufacturing by extracting fundamental information necessary for the particular stage. In this paper, methodology is proposed for generic feature extraction system from 3D CAD model cased on STEP format.

KEYWORDS: CAD, CAPP, STEP \& CAI
\end{abstract}

Received: Mar 13, 2020; Accepted: Apr 03, 2020; Published: Apr 30, 2020; Paper Id.: IJMPERDJUN202019

\section{INTRODUCTION}

For survival and rapid growth of the industry, competitive product pricing with the help of low production cost, increasing number of variants as well as rising demand for individual products[1], easy availability in the market, quick product modification or new product adoption according to market demand, etc. are some of the significant factors that play a crucial role. These factors can be optimized by integration as well as modification of different sections of manufacturing system such as Computer Aided Design (CAD), Computer Aided Manufacturing (CAM), Computer Aided Inspection (CAI) [2], Computer Aided Process Planning (CAPP), etc. CAPP is an important interface between CAD and CAM and integration of CAD and CAM is necessary to realize this interface. The system integration is achieved through the data exchange among different sections of the system. The starting stage of the product in manufacturing system is product design and the CAD models generated during design include extensive information about the product. The information in CAD file cannot be directly used for further stages of product manufacturing. Different CAD packages have their own proprietary data storage formats which require development of customized data extraction software to extract data from the CAD data file. To obviate this difficulty, neutral file formats like STEP and IGES have been developed which are compatible with all the CAD packages. The information in neutral file format can be used for various subsequent activities such as process planning, manufacturing, inspection, assembly planning, etc.

The advancement of CAPP has been slow than that reported for CAD database. Data extraction from CAD 
database and its use for downstream application development has been an important area of research, especially the required common information for subsequent activities of diverse CAD systems [3].

\section{LITERATURE REVIEW}

There has been a large amount of research in the area of feature recognition systems. Automated feature recognition has been an active research area in solid modelling for past decade and is believed to be a critical component for integration of CAD and CAM.

F. Zhou et al. [4] put forward an approach for integrating the set-up planning system with a feature-based CAD system for the computer-automated extraction of geometry and tolerance information. S. Q. Xie and X. Xu[5] have developed a prototype, STEP compliant process planning system for sheet metal product development. C. Panet al.[6] have put forward a fully automated assembly sequence planner, which directly extracts geometrical information from an input STEP file and then finds assembly sequences, which require a minimum or near-minimum number of assembly direction reorientations, for a given product design. X. Guan et al. [7] designed the lexical analyzer by using the object-oriented method and extracted the related information of features from the STEP file. The researchers converted the extracted information into an Attributed Adjacency Graph (AAG) and used the neural network to recognize the machining features. S. Sivakumara and V. Dhanalakshmi [2] used part features extracted from STEP file to integrate CAD, CAM and CAI.E. S. A. Nasar et al. [8] presented an automatic feature extraction system using object oriented approach. The researchers formulated logical rules based on geometric reasoning to recognize selected machining features.

Observing the literature, researchers has concluded that the data in STEP file can be used for further stages in manufacturing such as CAM, CAI, CAPP, etc. Manufacturing features are nothing but combination of different geometric features such as plane, cylindrical surface, toroidal surface, etc. So, the first step is to extract the geometric features of the 3D part model. Researchers have decided to use the STEP file to extract the part geometric features.

Figure 1 illustrates the flow diagram of the methodology implemented in the present work. It consists of four major steps which are listed as follows:

- Generate a CAD Model for the part.

- Create a STEP file from CAD Model.

- Extract geometric features data from the STEP file.

- Save geometric features data in a file.

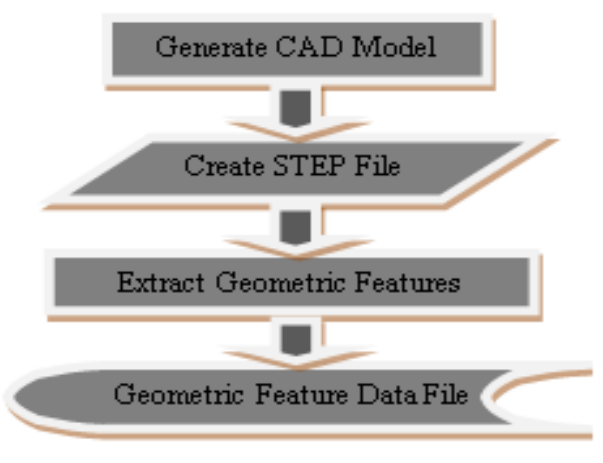

Figure 1: Methodology Implemented 


\section{INFORMATION EXTRACTION FROM STEP FILE}

\subsection{Generate CAD Model}

Three types of CAD models have been widely used by the researchers for part modelling, i.e., wire-frame model, surface model, and solid model. Parts are represented by points, lines and curves in wire-frame models. In surface model, parts are represented by their bounding faces and each face has edges and vertices on it. Surface models provide a better graphic interface than wire-frame models. A solid model is a superior approach for creating 3D models. Constructive Solid Geometry (CSG) and Boundary Representation (B-rep) methods find wide acceptance among the researchers for part modelling in CAPP [9]. This has made authors to think use of 3D CAD model suitable for part modelling.

In this paper, 3D CAD Model for a prismatic part as shown in Figure 2 is generated using two different CAD systems, namely CATIA and CREO. The CAD Model selected has eight features such as plain surface, through cylindrical hole, rectangular slot, semicircular slot, edge chamfer, edge rounding, rectangular blind hole/depression and odd shaped blind hole/depression.

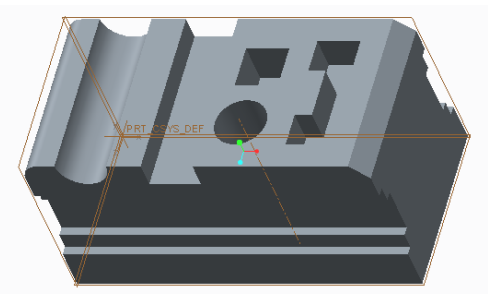

Figure 2: CAD Model

\subsection{STEP File}

STEP (Standard for Exchange of Product data) is the precise computer interpretable representation of product data, which offers the resource of an efficient information exchange between product design and different manufacturing activities as well as it provides compatibility with different CAD systems. It uses the data modelling language EXPRESS to specify the product information to be represented that enables precision and consistency of representation. A part of STEP file is shown in figure 3 .

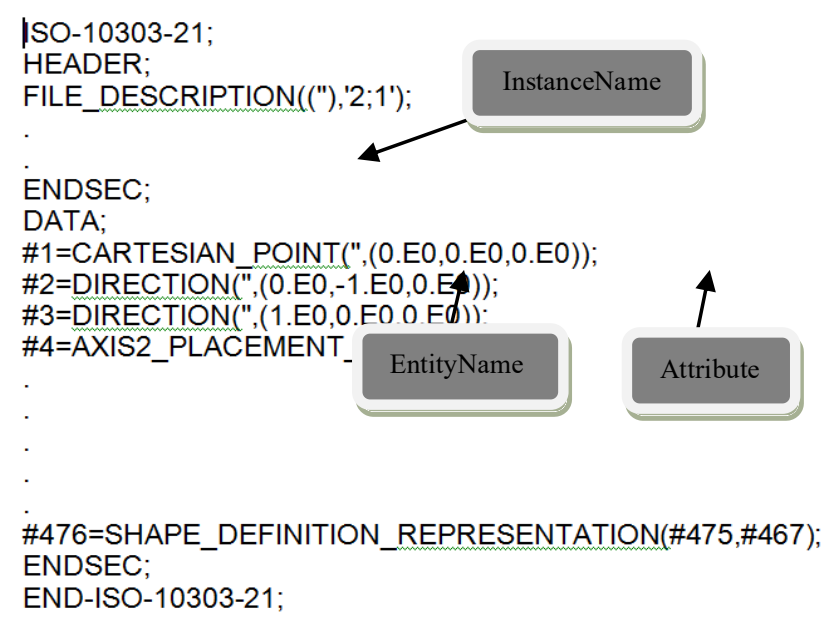

Figure 3: STEP File.

The STEP file starts with ISO-10303-21 and ends with END-ISO-10303-21. It is divided into two distinct sections which 
are header section and a data section.

\subsubsection{Header section}

The header section begins with the key word "HEADER" and ends with "ENDSEC" while the data section begins with "DATA" and ends with "ENDSEC". The header section includes information such as file name, date, CAD software used, etc.

\subsubsection{Data Section}

The DATA section is the most important part of STEP file. It includes a number of correlated or independent statements defined by EXPRESS. These statements describe different components of drawing such as face, edge, point, etc. The statement in the data section is defined by Wirth Syntax Notation (WSN). WSN is a meta-syntax, that is, a formal way to describe formal languages, in which a series of entities are used to describe the information product. The structure of sentences is as follows:

\#Instance Name = Entity Name (Attribute 1, Attribute 2, . .);

The Instance Name is a positive integer used to represent the statement number starting from one and continues in sequence up to the last statement. The Entity Name is a self illustrative reserved word such as ADVANCED_FACE, ORIENTED_EDGE, PLANE, LINE, CARTESIAN_POINT, CYLINDRICAL_SURFACE, etc., which gives information about different geometrical features of the product. The attribute may be referring to instance name, value, string, coordinates of a point, etc.

\subsubsection{Data structure}

The product data stored in data section have a hierarchical arrangement of data elements, as shown in Figure 4. For every component drawing, there will be single statement with entity name CLOSED_SHELL containing attributes as instance names for the entity ADVANCED_FACE equal to the total bounding surfaces of the component which further give reference to instance names for FACE_OUTER_BOUND and surface entity such as CYLINDRICAL_SURFACE or CONICAL_SURFACE or TORROIDAL_SURFACE or PLANE, etc. The first attribute in the statement for surface entity will further give reference to the instance name of the entity AXIS2_PLACEMENT_3D and other attributes will give dimensional information about the surface. The first attribute in AXIS_PLACEMENT_3D give reference to the entity name of entity CARTESIAN_POINT and other two attributes give references to entity names for entity DIRECTION. Here, the axis of surface entity in 3D space is defined by a point and two directions. The attribute in the statement for entity FACE_OUTER_BOUND will give reference to the instance name of entity EDGE_LOOP which further give reference to the set of instance names of entity ORIENTED_EDGE. The oriented edge may be a semicircle or a straight line. There will be a single attribute in each statement for ORIENTED_EDGE which provide reference to instance name for entity EDGE_CURVE. First two attributes in the entity EDGE_CURVE give reference to entity VERTEX_POINT defining start and end point of the basic drawing entity and the third attribute give reference to the basic drawing entity i.e. LINE or CIRCLE. Attributes in a statement for LINE will further give reference to entity names CARTESIAN_POINT and VECTOR. The attributes in entity CARTESIAN_POINT will give start point or midpoint of the line while the first attribute in the entity. VECTOR will give reference to entity name for entity DIRECTION where we get the direction of the line vector and the second attribute gives the length of the line vector. First attribute in the statement for CIRCLE will give reference to entity name for AXIS_PLACEMENT_3D where we get data related to the axis of the circle while second 
attribute will give circle radius. The first attribute in AXIS_PLACEMENT_3D give reference to the entity name of entity CARTESIAN_POINT and other two attributes give references to entity names for entity DIRECTION. Here the axis of CIRCLE in 3D space is defined by a point and two directions.

Thus, we can obtain information about the solid model in the form of bounding surfaces and their axis in 3D space. The bounding surface consists of the edges defining the boundary of the surface as well as edges inside the boundary on the surface. Straight line and curved line are two types of edges present on the surface. A Line is defined by a start point, end point, line vector, vector start point and length as well as direction. While a circle is defined by a start point, end point, radius, centre point and axis of the circle.

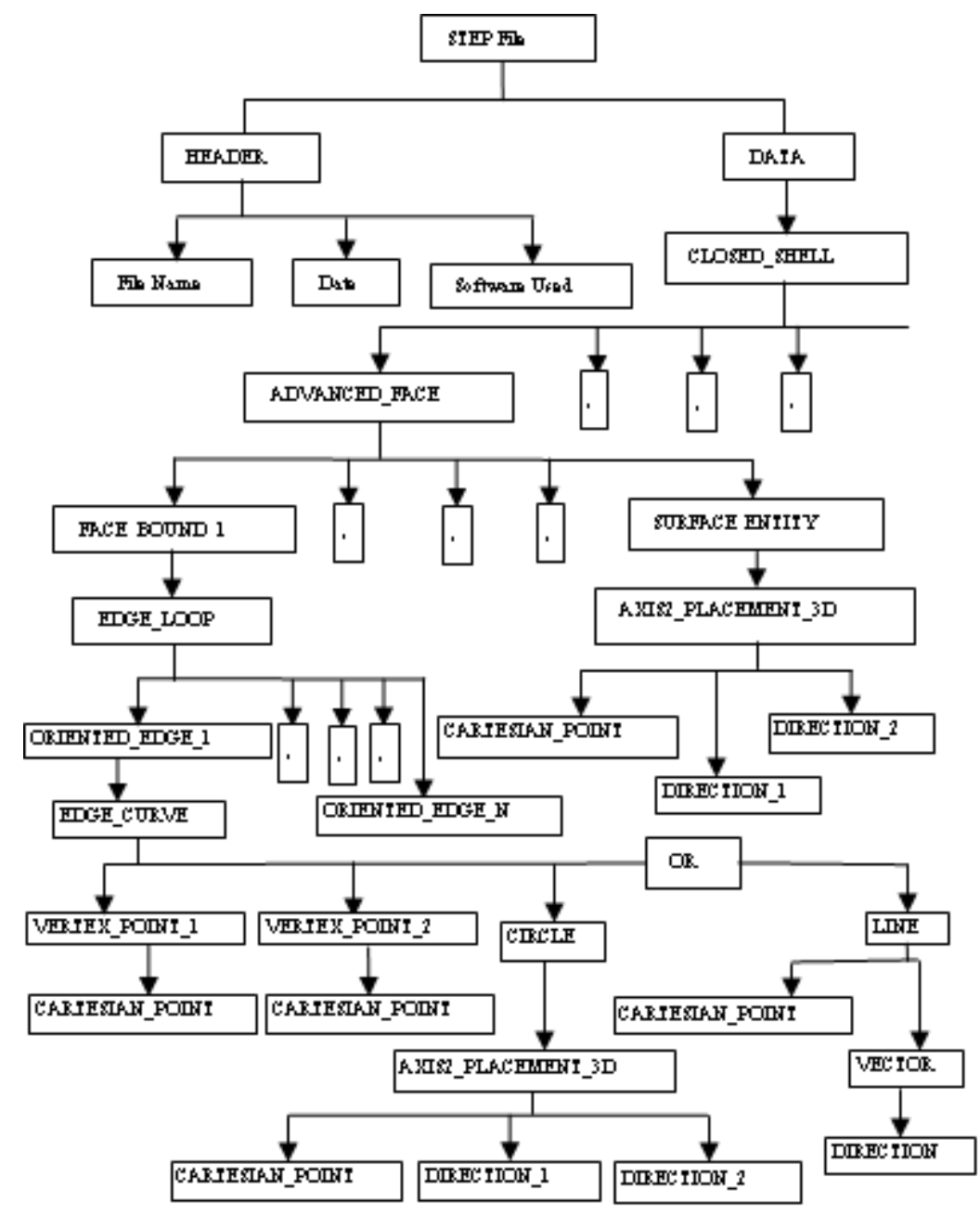

Figure 4: STEP File Structure

\section{GEOMETRIC FEATURE EXTRACTION}

The feature extraction from STEP file is nothing but to extract geometry and topography information of the 3D model from data section. In this paper, Visual $\mathrm{C}^{++}$programming language is used to extract feature information from STEP file. Sequential search approach is used to scan the file to search the required feature data of the component. At the start, program search for the key word CLOSED_SHELL in every statement of the STEP file and identify the unique statement containing this key word. The attributes in this statement give links relating to entity ADVANCED_FACE which gives further links for the data related to different bounding surfaces of the object. The data about start point, end point, length 
and direction of lines; centre point, radius and the axis of circles on the bounding surfaces are obtained by searching the statements in hierarchy obtained from the links provided by the attributes of the different entities in hierarchy from ADVANCED_FACE. This is the basic information necessary for different manufacturing activities, which are stored in memory as STEP file format, which provides information for feature recognition. The process of feature information extraction flowchart is shown in Figure 5.

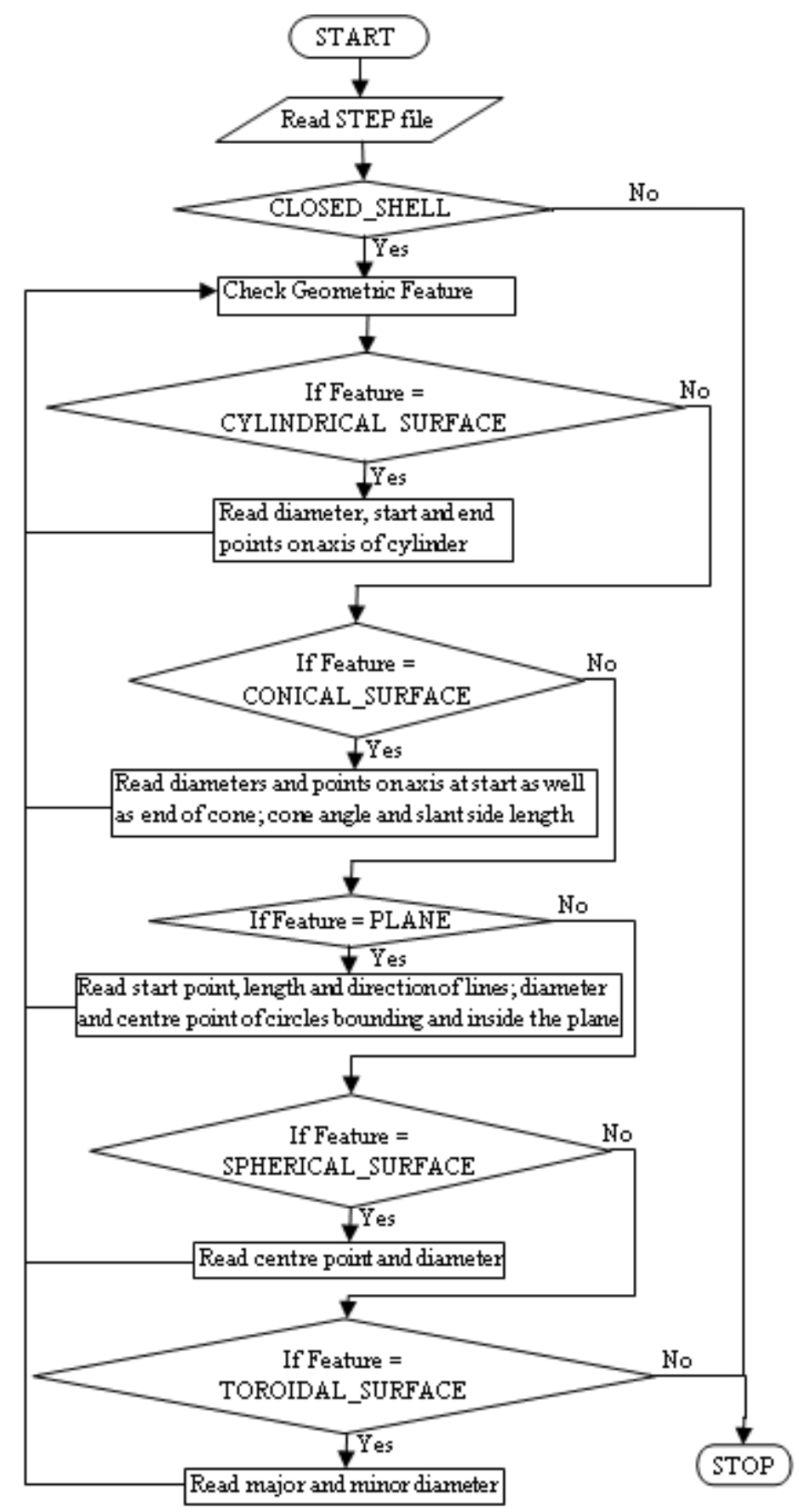

Figure 5: Feature Extraction Flowchart

The geometrical and topological information of component is available in the STEP file for the 3D part solid model. Hence, the features extracted from STEP files give information regarding size, shape and location of different surfaces of the finished component in $3 \mathrm{D}$ space which are nothing but the geometric features. The geometric features may 
be plane, cylindrical surface, conical surface, spherical surface, etc.

A STEP file is created for the CAD model, which is shown in Figure 2 and this STEP file is used as an input to the STEP reader. Step reader is a computer program written in Visual $\mathrm{C}++$ which reads part geometric information in STEP file and output of a program stored in the separate output file of the feature recognition system. This output gives the information about component geometries such as plane, cylindrical surface, conical surface, etc. A part of the output file is shown in Figure 6.

\author{
Number of ADCANCED_FACES = 46 \\ ADCANCED_FACE [1] \\ BOUNDING SURFACE TYPE IS 'PLANE' \\ [1] BONDING_EDGE_LOOP = FACE_OUTER_BOUND \\ Number of Oriented Edges $=4$ \\ LINE 1 VORTEX_POINT $1=0 . \mathrm{E} 0,2.5 \mathrm{E} 1,0 . \mathrm{E} 0$ \\ LINE 1 VORTEX_POINT $2=2 . E 2,2.5 E 1,0 . E 0$ \\ LINE 1 VECTOR START POINT = 0.E0,2.5E1,0.E0 \\ LINE 1 VECTOR DIMENSION $=2 . \mathrm{E} 2$ \\ LINE 1 VECTOR DIRECTION $=1 . \mathrm{E} 0,0 . \mathrm{E} 0,0 . \mathrm{E} 0$
}

LINE 4 VORTEX_POINT $1=2 . E 2,0 . E 0,0 . E 0$

LINE 4 VORTEX_POINT $2=2 . \mathrm{E} 2,2.5 \mathrm{E} 1,0 . \mathrm{E} 0$

LINE 4 VECTOR START POINT = 2.E2,0.E0,0.E0

LINE 4 VECTOR DIMENSION $=2.5 \mathrm{E} 1$

LINE 4 VECTOR DIRECTION $=0 . \mathrm{E} 0,1 . \mathrm{E} 0,0 . \mathrm{E} 0$

ADCANCED_FACE [14]

BOUNDING SURFACE TYPE IS 'CYLINDRICAL_SURFACE'

[1] BONDING_EDGE_LOOP = FACE_OUTER_BOUND

Number of Oriented Edges $=4$

CIRCLE 1 VORTEX_POINT $1=1.35 E 2,0 . E 0,7 . E 1$

CIRCLE 1 VORTEX_POINT $2=1.05 E 2,0 . E 0,7 . E 1$ 


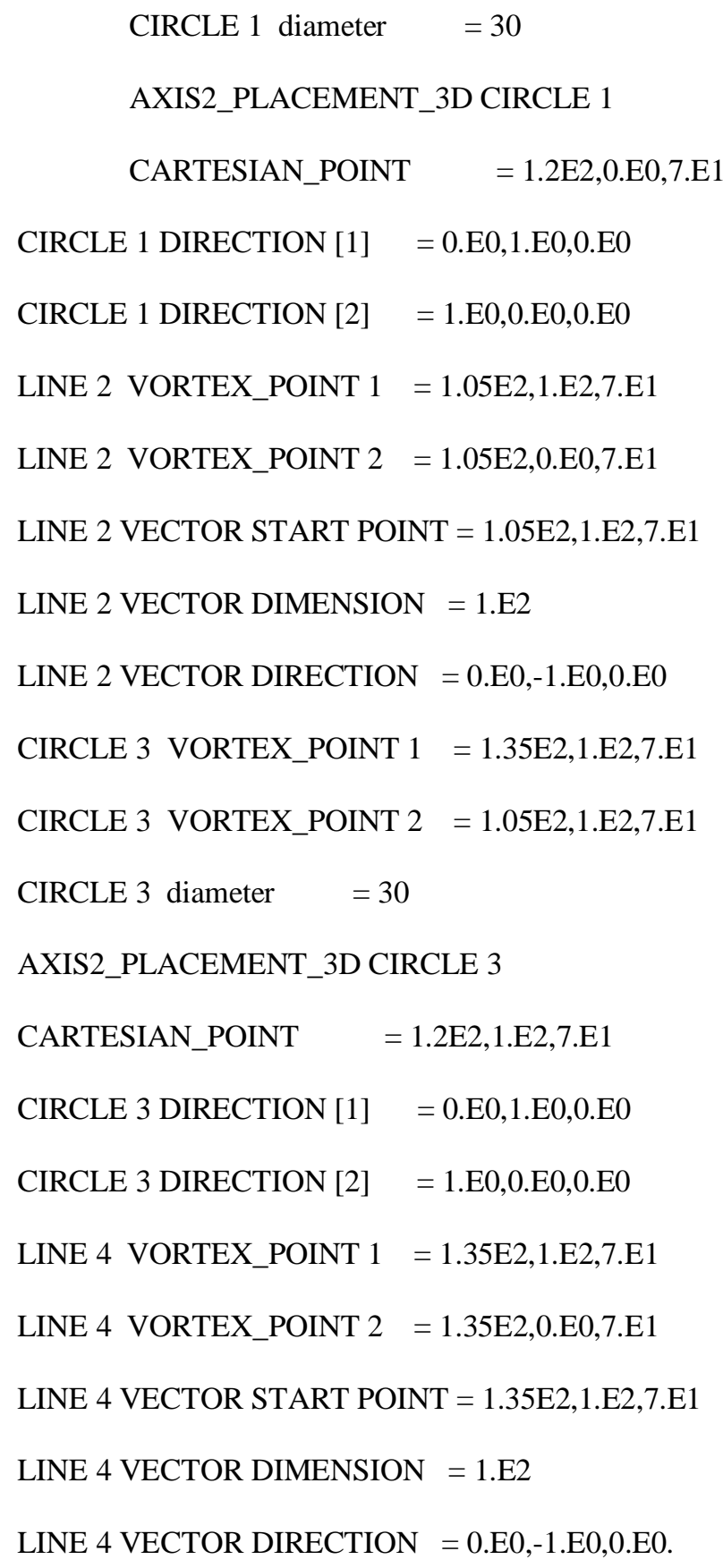

Figure 6: Program Output

\section{RESULTS AND DISCUSSIONS}

The STEP reader developed examines the STEP file and the output is obtained in the form of information of the geometries of the component in 3D space. In case of plane, the information about different entities on the plane such as line or circle and their exact location on the plane is extracted. In case of line the coordinates of end points, the line vector start point, vector dimension and direction are extracted. The circle is divided into two semicircles and in the case of each semicircle the coordinates of two end points on the semi circle, diameter and its axis placement in 3D space is extracted. The cylindrical surface is divided in two halves and information about two semicircles at the end of each half and two lines joining the semicircles is extracted. The conical surface is also divided in two halves and information about two semicircles 
at the end of each half, cone angle, cone slant side length and two lines joining the semicircles is extracted.

\section{CONCLUSIONS}

In this paper, an attempt has been made to extract the information related to different geometries of components using a STEP file created from the CAD model. A generic STEP reader is developed using programming language, Visual C++ and tested for different CAD models developed from PROE and CATIA to obtain and store information related to geometric features of the 3D CAD model. The output of a STEP reader can be used as input to Computer Aided Inspection or Computer Aided Process Planning or Computer Aided Assembly Planning.

\section{REFERENCES}

1. A Hees and G Reinhart, (2015), “Approach for Production Planning in Reconfigurable Manufacturing Systems “, 9th CIRP Conference on Intelligent Computation in Manufacturing Engineering, Vol.33, pp. 70 - 75.

2. S. Sivakumar and V. Dhanalakshmi, (2013), "An approach towards the integration of CAD/CAM/CAI through STEP file using feature extraction for cylindrical parts", International Journal of Computer Integrated Manufacturing, Vol. 26, No. 6, pp. 561 570.

3. T Yifei et al.,(2008), “A feature-extraction-based process-planning system”, International Journal of Advanced Manufacturing Technology, Vol. 38, pp. 1192-1200.

4. F. Zhou et al.,(2002), "Form Feature and Tolerance Transfer from a 3D Model to a SetupPlanning System", International Journal of Advanced Manufacturing Technology, Vol. 19, pp. 88-96.

5. S. Q. Xie and X. Xu,(2006), “A STEP-compliant process planning system for sheet metal parts”, International Journal of Computer Integrated Manufacturing, Vol. 19, No.6, pp. 627-638.

6. C. Pan et al., (2006), “Automatic assembly sequence planning from STEP CAD files”, International Journal of Computer Integrated Manufacturing, Vol. 19, No. 8, pp. 775-783.

7. X. Guanet al., (2010), "Machining feature recognition of part from STEP file based on ANN," 2010 International Conference on Computer, Mechatronics, Control and Electronic Engineering, Changchun, pp. 54-57.

8. E. S. A. Nasret al., (2014), “A Feature Recognition System using Geometric Reasoning”, International Conference on Manufacture of Lightweight Components - ManuLight2014, pp. 238-243.

9. P. K. Jain and Sharvan Kumar, (1998), “Automatic Feature Extraction in PRIZCAPP”, International Journal of Computer Integrated Manufacturing, Vol. 11, No. 6, pp. 500-512.

10. D. L. Gunapala, O. Gunapala \& S. Egodage, “Application of Modeling and Simulation Tools for Design of Plastic Products”, International Journal of General Engineering and Technology (IJGET), Vol. 8, Issue 1, pp. 1-8

11. Viswa Mohan Pedagopu \& Manish Kumar, "The Conventional Extraction of Features from Intricate Prismatic Part for Computer Aided Process Planning System (CAPPS)", International Journal of Mechanical Engineering (IJME), Vol. 2, Issue 4, pp. $51-58$

12. Bishnu M Jha \& A Mandal, "Overcut and Profile of the Machined Features in Electrochemical Machining”, IASET: International Journal of Metallurgical, Materials and Chemical Engineering (IASET: IJMMCE), Vol. 1, Issue 1, pp. 29-40 


\section{AUTHOR'S PROFILE}

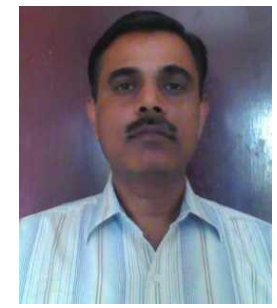

C. S. GHADAGE, Lecturer, Mechanical Engineering Department, Govt. Polytechnic, Pune, Dist: Pune-411016, India, csghadge@gmail.com

Dr. B. S. PABLA, Professor, Mechanical Engineering Department, National Institute of Technical Teachers' Training and Research, Chandigarh-160019, India, drbspabla@gmail.com

Dr. S. S. DHAMI, Professor, Mechanical Engineering Department, National Institute of Technical Teachers' Training and Research, Chandigarh-160019, India, ssdhami@yahoo.com 